\title{
30 Jahre Berner Konvention
}

\section{Jochen Schumacher}

\section{(C) Springer-Verlag 2009}

Neben der Vogelschutzrichtlinie und der Bonner Konvention (Convention on the Conservation of Migratory Species of Wild Animals - CMS) besteht auch die Berner Konvention seit 30 Jahren.

Das „Übereinkommen über die Erhaltung der europäischen wildlebenden Pflanzen und Tiere und ihrer natürlichen Lebensräume“ (Berner Konvention) zählt zu den bedeutenden Konventionen zum Schutz von Flora und Fauna und will einen Beitrag zur Bewahrung des europäischen Naturerbes erbringen.

Das Berner Übereinkommen wurde am 19.9.1979 in Bern in der Schweiz unterzeichnet und trat am 1.7.1982 in Kraft. Vertragsparteien sind die jeweiligen Mitglieder des Europarates. Bisher sind dem Übereinkommen 45 Staaten beigetreten, darunter auch europäischen Staaten außerhalb der Europäischen Union und vier afrikanische Staaten: Burkina Faso, Marokko, Senegal, Tunesien.

Die Ziele der Konvention sind

- die Schaffung eines Mindestschutzes für die meisten wild lebenden Pflanzen- und Tierarten und ihrer natürlichen Lebensräume sowie

- ein intensiver Schutz für besonders bedrohte Tier- und Pflanzenarten - vor allem der wandernden Arten.

Die Vertragsparteien müssen die erforderlichen Maßnahmen ergreifen, um die Population der wild lebenden Pflanzen und Tiere auf einem Stand zu erhalten oder auf einen Stand zu bringen, der insbesondere den ökologischen, wissenschaftlichen und kulturellen Erfordernissen entspricht.

Die Berner Konvention sieht im Gegensatz zu vielen anderen internationalen Konventionen vor, dass die Vertragsstaaten die Pflicht haben, geeignete Maßnahmen zum Schutz der Lebensräume der in den Anhängen I und II aufgeführten Tier- und Pflanzenarten zu ergreifen. Nach Art. 4 der Berner Konvention hat der Vertragsstaat seine besondere Aufmerksamkeit dem Schutz der für wandernde Arten bedeutsamen Gebiete zu widmen. Die Berner Konvention enthält - im Interesse einer wirksamen Sicherung der von der Richtlinie erfassten Arten - detaillierte $\mathrm{Zu}-$ griffs-, Störungs- und handelbezogene Verbote.

Ass. Jur. Jochen Schumacher,

Redaktion Natur und Recht,

Tübingen, Deutschland
Nach der Berner Konvention müssen die Vertragsstaaten:

- Die notwendigen Schritte unternehmen, um eine nationale Politik zur Erhaltung wild lebender Pflanzen und Tiere zu fördern;

- bei der Planungs- und Entwicklungspolitik sowie bei Maßnahmen gegen die Umweltverschmutzung die Erhaltung wild lebender Pflanzen und Tiere zu berücksichtigen;

- die Förderung der Öffentlichkeitsarbeit in Bezug auf die Notwendigkeit wild lebende Pflanzen- und Tierarten sowie ihre Lebensräume zu erhalten;

- Gebiete, die für wandernde Arten von Bedeutung sind und die als Überwinterungs-, Sammel-, Futter-, Brutoder Mauserplatz dienen, ihre besondere Aufmerksamkeit zukommen zu lassen.

Die Konvention unterscheidet zwischen

- „streng geschützten“ Arten in Anhang I (Pflanzen) und Anhang II (Tiere) und

- den in Anhang III ,,geschützten“ Tierarten.

- Anhang IV listet verbotene Mittel und Methoden zum Töten und Fangen auf.

Für derzeit rund 500 streng geschützte Pflanzenarten ist das Pflücken, Sammeln, Abschneiden, Ausgraben oder Ausreißen sowie, soweit erforderlich, auch der Besitz oder der Verkauf dieser Arten zu verbieten; ihre Lebensräume sollen zudem geschützt werden.

Rund 600 Tierarten sind derzeit streng geschützt. Für diese Tierarten ist unter anderem jede Form des absichtlichen Fangens, Haltens und Tötens sowie das mutwillige Beschädigen oder Zerstören von Brut- oder Raststätten zu verbieten.

„Geschützte“ Tierarten dürfen grundsätzlich genutzt werden, es sind jedoch Art und Ausmaß der Nutzung vorzuschreiben. Mittel und Methoden des Fangens und Tötens sowie die Nutzungsformen werden aufgelistet, die an den ,geschützten“ Tierarten nicht angewendet werden dürfen.

Für die Mitgliedstaaten der Europäischen Union wurden die rechtlichen Aspekte des Artenschutzes der Berner Konvention weitgehend in die FFH-Richtlinie übernommen. Insoweit dient die FFH-Richtlinie der Umsetzung der Verpflichtung aus der Berner Konvention. Die Berner Konvention hat aber auch weiterhin z.B. für die Umsetzung der Biodiversitätskonvention auf europäischer Ebene und für lokale Aktivitäten große Bedeutung. 